\title{
Decompressive Surgery for Craniovertebral Foramen Magnum Stenosis with Medullary Compression in Paediatric Skeletal Dysplasia Syndromes
}

\author{
Cristiano Antunes $^{1^{*}}$ (D), Paulo Pereira ${ }^{2,3}$ (iD, Daniela Alves ${ }^{4}$ (D) Rui Vaz ${ }^{1,3}$ (i) and Josué Pereira ${ }^{3,5}$ (iD \\ ${ }^{1}$ Department of Neurosurgery, Centro Hospitalar Universitário de São João, Porto, Portugal \\ ${ }^{2}$ Department of Neurosurgery, Centro Hospitalar Universitário de São João, Neurosurgical Spine Unit, \\ Porto, Portugal \\ ${ }^{3}$ Faculty of Medicine, University of Porto, Portugal \\ ${ }^{4}$ Department of Paediatrics, Centro Hospitalar Universitário de São João, Porto, Portugal \\ ${ }^{5}$ Department of Neurosurgery, Centro Hospitalar Universitário de São João, Neurosurgical Paediatric Unit, \\ Porto, Portugal
}

*Corresponding author: Cristiano Martins Antunes, Department of Neurosurgery, Centro Hospitalar Universitário de São João, Porto, Portugal, Serviço de Neurocirurgia, Centro Hospitalar Universitário de São João, Alameda Prof, Hernâni Monteiro, 4200-319 Porto, Portugal, Tel: +351-9193-95519

\begin{abstract}
Background: More than 450 skeletal dysplasias have been described including Achondroplasia. Due to defective ossification of the occipital bone and upper cervical spine, neural structures may be compressed, causing increased morbidity/mortality during childhood, mainly from sleep apnoea and other respiration abnormalities, myelopathy, syringomyelia, hydrocephalus, and even sudden infant death. This highlights the importance of early and effective treatment of craniovertebral stenosis in infants with skeletal dysplasias.
\end{abstract}

Cases Description: Reports of two paediatric patients with skeletal dysplasia treated at a very young age by decompressive surgery for craniovertebral stenosis with cervicomedullary compression - a four-month old achondroplastic infant and a 13-month-old infant with skeletal dysplasia. Both patients presented neurological signs from cervicomedullary compression. Multimodal neuromonitoring and ultrasound were applied intraoperatively. Immediate postoperative period was uneventful, and both patients had a favourable neurological evolution during follow up. Surgical procedures, pre and postoperative status are described. A brief review of pertinent literature is discussed.

Conclusion: Cervicomedullary compression at the craniovertebral junction is common in patients with skeletal dysplasia. All infants with skeletal dysplasias who may have an abnormal craniocervical junction with neural compression must undergo neuroimaging and polysomnography studies. Surgery at this age has some specificities and, in our view, the intraoperative multimodal neuromonitoring and ultrasound evaluation are helpful tools which may improve surgical success. Early diagnosis and referral to specialized centres are of paramount importance since timely treatment maximizes the neurological outcome improving the natural history.

\section{Keywords}

Osteochondrodysplasias, Achondroplasia, Craniovertebral junction, Foramen magnum, Spinal cord compression

\section{List of Abbreviations}

AChP: Achondroplasia; CSF: Cerebral spinal fluid; MRI: Magnetic resonance imaging

\section{Introduction}

Skeletal dysplasias are heritable disorders with over 450 described entities affecting primarily cartilage and bone [1]. Phenotypes are widely variable and clinical diagnosis can be challenging. In the most severe forms, skeletal dysplasias are associated with several neurological complications such as macrocephaly,

Citation: Antunes C, Pereira P, Alves D, Vaz R, Pereira J (2021) Decompressive Surgery for Craniovertebral Foramen Magnum Stenosis with Medullary Compression in Paediatric Skeletal Dysplasia Syndromes. Neurosurg Cases Rev 4:073. doi.org/10.23937/2643-4474/1710073

Accepted: July 21, 2021; Published: July 23, 2021

Copyright: (c) 2021 Antunes C, et al. This is an open-access article distributed under the terms of the Creative Commons Attribution License, which permits unrestricted use, distribution, and reproduction in any medium, provided the original author and source are credited. 
hydrocephalus, developmental delay, foramen magnum and/or spinal stenosis, with brainstem, spinal cord and nerve roots compression. Advances in genetic testing has had importance in establishing the diagnosis in some affected patients. This is key not only for genetic counselling but also to expand our knowledge on genotype-phenotype correlation, which in its turn may allow a better risk prediction and personalised interventions to achieve better outcomes in these patients [2].

Achondroplasia (AChP) is the most common cause of skeletal dysplasia and short-limbed dwarfism [2,3]. AChP incidence is 1 per 15,000-40,000 live births with an autosomal dominant inheritance pattern [4]. It affects the size and shape of the skull, trunk and limbs [5]. It is caused by a gain-of-function mutation on Fibroblast Growth Receptor 3 resulting in a defective endochondral bone formation. As a result, a malformative cartilage undergrowth prevents a proper endochondral ossification. Neonates with AChP present a disproportionately short humerus and femur (rhyzomelia). The phenotype is characterized by short stature with marked shortening of the limbs.

In this paper, we aim to highlight the importance of early screening for craniovertebral stenosis in infants with skeletal dysplasias, to proceed to a timely referral to Neurosurgical evaluation. Additionally, a brief revision of the subject is performed. We report two paediatric patients with skeletal dysplasia treated at a very young age by decompressive surgery for craniovertebral stenosis with cervicomedullary compression associated with skeletal dysplasia.

\section{Background}

The craniovertebral junction is a high mobility range area composed of the occipital bone and cervical $\mathrm{C} 1$ and $\mathrm{C} 2$ vertebrae enclosing the foramen magnum at the point the rhombencephalon and spinal cord connect in the cervicomedullary junction. It is an anatomically complex area between the skull and the spinal column where the transition between intramembranous and endochondral ossification takes place during embryogenesis. Impaired endochondral ossification in occipital bone and spine, as occurs in skeletal dysplasias such as AChP, can cause osseous underdevelopment with small foramen magnum size and upper cervical spinal canal stenosis, compressing the cervicomedullary transition, vertebral and spinal arteries and spinal roots of the accessory nerves [6,7]. A prospective study conducted in AChP infants found radiological evidence of craniovertebral stenosis in $58 \%$ of patients, $35 \%$ of them with cervicomedullary compression [8]. Direct compression and injury on the cervicomedullary junction cause myelopathy, syringomyelia and hydrocephalus. Additionally, chronic compression on vertebral arteries can produce hypoxic damage to respiratory centres leading to an impairment of respiratory control, which results in sleep apnoea syndrome (and other respiration abnormalities) and sudden infant death [9]. This is accounted for as the main cause of mortality in these patients during childhood [10]. Simmons, et al. published a review which showed a mortality rate of 41.4 per 1000 in patients with AChP during their first year of life [11], which reinforces the importance of carefully investigating and treating craniovertebral stenosis in patients with AChP, as well as other infants with skeletal dysplasias with similar craniovertebral compression.

\section{Clinical Presentation, Diagnosis \& Management}

Diagnosing cervicomedullary compression requires a thorough anamnesis, emphasizing respiratory symptoms, as well as physical examination, sleep studies and neuroimaging, especially Magnetic Resonance Imaging (MRI) T2-weighted sequences in the sagittal plane. Cervicomedullary compression presentation includes hypotonia, upper or lower extremity paresis, apnoea, hyperreflexia or hypertonia, and development delay in motor skills.

According to the American Academy of Paediatrics Guidelines (2005) [12], all infants with AChP should undergo neuroimaging with Computed tomography (CT) or MRI and polysomnography [13]. White matter injury in the brainstem and spine cord can be appreciated as a high-intensity T2-weighted signal and diffusion tensor imaging reveals disruption of white matter tracts [14].

Currently, children with AChP continue to be underscreened for foramen magnum stenosis. Nadel JL, et al. [12] reviewed 3577 children with AChP and found that only $41.9 \%$ had done some sort of screening and only $13.9 \%$ were properly studied according to American Academy of Paediatrics Guidelines (2005) [12].

The decision to perform foramen magnum decompression is made based on stenosis degree, neural structure compression - with/without myelomalacia - and the presence of clinical symptoms and signs. Intervention is required if there is clinical evidence of brain stem and/or upper spinal cord compression from foramen magnum stenosis and an otherwise unexplained developmental disorder or respiratory anomaly [7]. These cases require an early and aggressive treatment to avoid medulla oblongata and high cervical spine cord myelopathy which are linked to a higher risk for sudden death by central respiratory failure $[15,16]$. Kubota T, et al. published a literature review and provided clinical guidelines to assist the decision-making process in several aspects of patients with AChP [17]. According to these guidelines, foramen magnum decompression is recommended for managing neural compression due to foramen magnum stenosis associated with neurological symptoms, abnormal neurological findings, and central respiratory disorders (Recommendation grade 1, evidence level B) [17]. 


\section{Prognosis, Outcomes and Risks}

Surgical foramen magnum decompression demonstrated a significant and sustained improvement in neurological and respiratory function when it is combined with ventilatory therapies if needed for possible respiratory compromise $[8,18]$. Possible surgical complications include cerebral spinal fluid (CSF) leakage, infection, pseudomeningocele and venous sinus haemorrhage [19-23].

\section{Cases Presentation}

\section{Case 1}

A caucasian male with prenatal diagnosis of AChP was born after a full-term pregnancy from young healthy parents. There was no familiar history of skeletal dysplasia. At four months of age, he was referred to our department after a one-month evolution of cervical hyperextension and failure to thrive, with crossing of two percentile lines downwards in weight and length, at the time growing under the 5th percentile. On neurological examination, he presented opisthotonos, slight quadriparesis, and poor head control. He had no signs of intracranial hypertension. Skeletal X-rays revealed a large cranium, with short base and a small foramen magnum, flat vertebral bodies, small iliac bones, and proximal shortening of long bones. MRI of the craniovertebral junction showed a marked cervicomedullary compression due to foramen magnum stenosis with high signal intensity changes on T2weighted images as shown in Figure 1.
Surgery was performed through a suboccipital craniectomy of approximately $2 \times 2$ centimetres and C1 laminectomy with cervicomedullary decompression. Multimodal intraoperative neuromonitoring was used to maximize surgical safety (shown in Figure 2). After decompression, an increase in the amplitude of motor evoked potentials waves was observed. Intraoperative ultrasound imaging was used to assess cervicomedullary decompression. Duraplasty was not required as a good expansion of CSF spaces was obtained and CSF flow velocities improved after bone decompression and posterior atlanto-occipital membrane dissection.

The patient was admitted in the paediatric Intensive Care Unit for two days after surgery. The postoperative period was uneventful. There were no respiratory complicationsand opisthotonos resolution wasobserved. A postoperative MRI showed a good cervicomedullary decompression (Figure 3). Good clinical outcome was observed during follow up. Seven-years after surgery the child presents no neurological impairment (mRankin scale grade 1) nor any respiratory problems (Figure 4).

\section{Case 2}

A caucasian male was born by caesarean dystocic delivery at 41 weeks after an uneventful pregnancy. There was no previous familiar history of skeletal dysplasia nor other hereditary diseases. He presented postpartum jaundice which was treated with phototherapy for four days. In addition, he had a patent ductus arteriosus without hemodynamic repercussion and did not require intervention. At five months, he

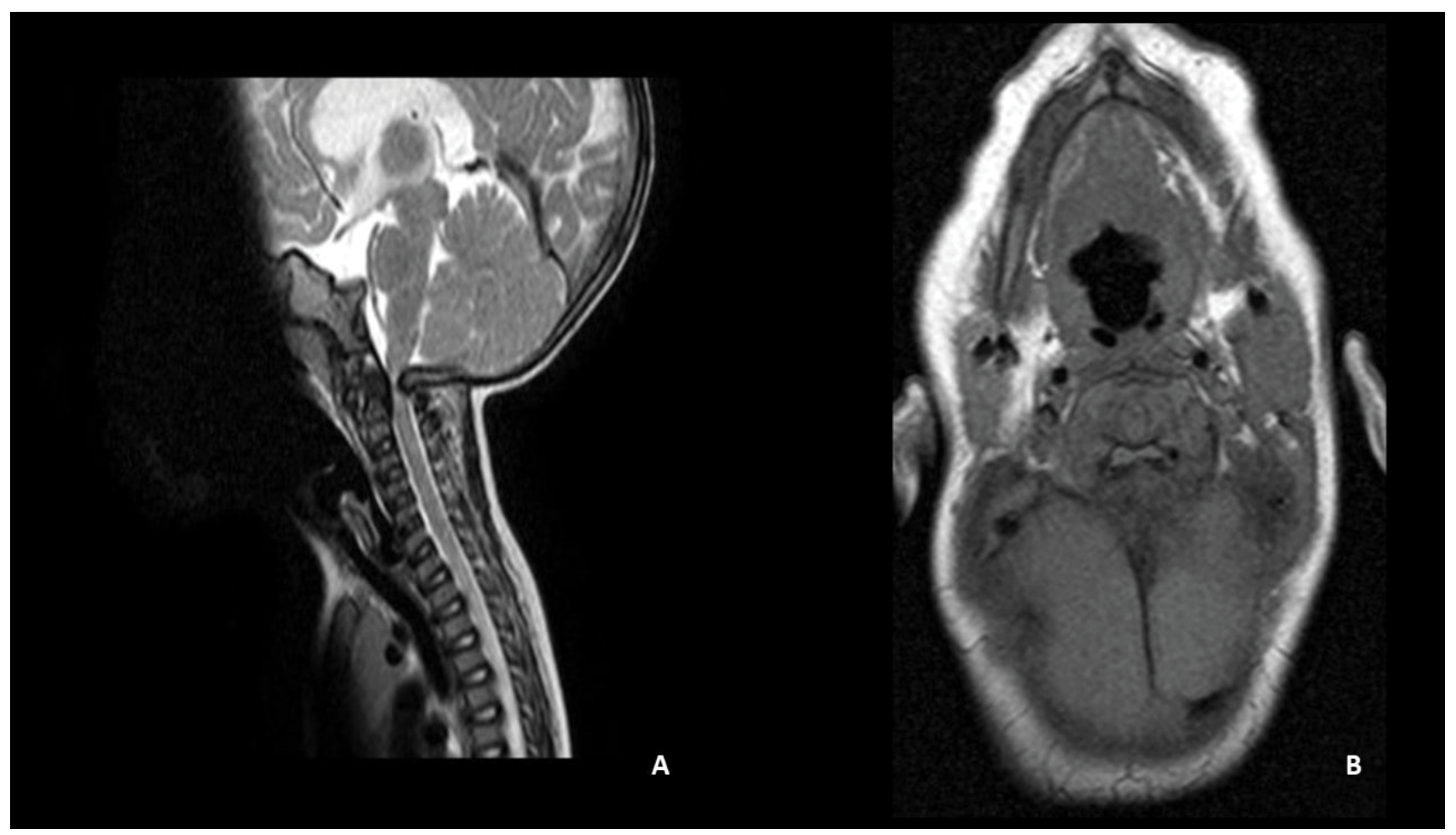

Figure 1: Patient 1- MRI showing the craniovertebral transition in sagittal (A) Axial; B) Planes. A marked compression in the cervicomedullary transition is observed. 


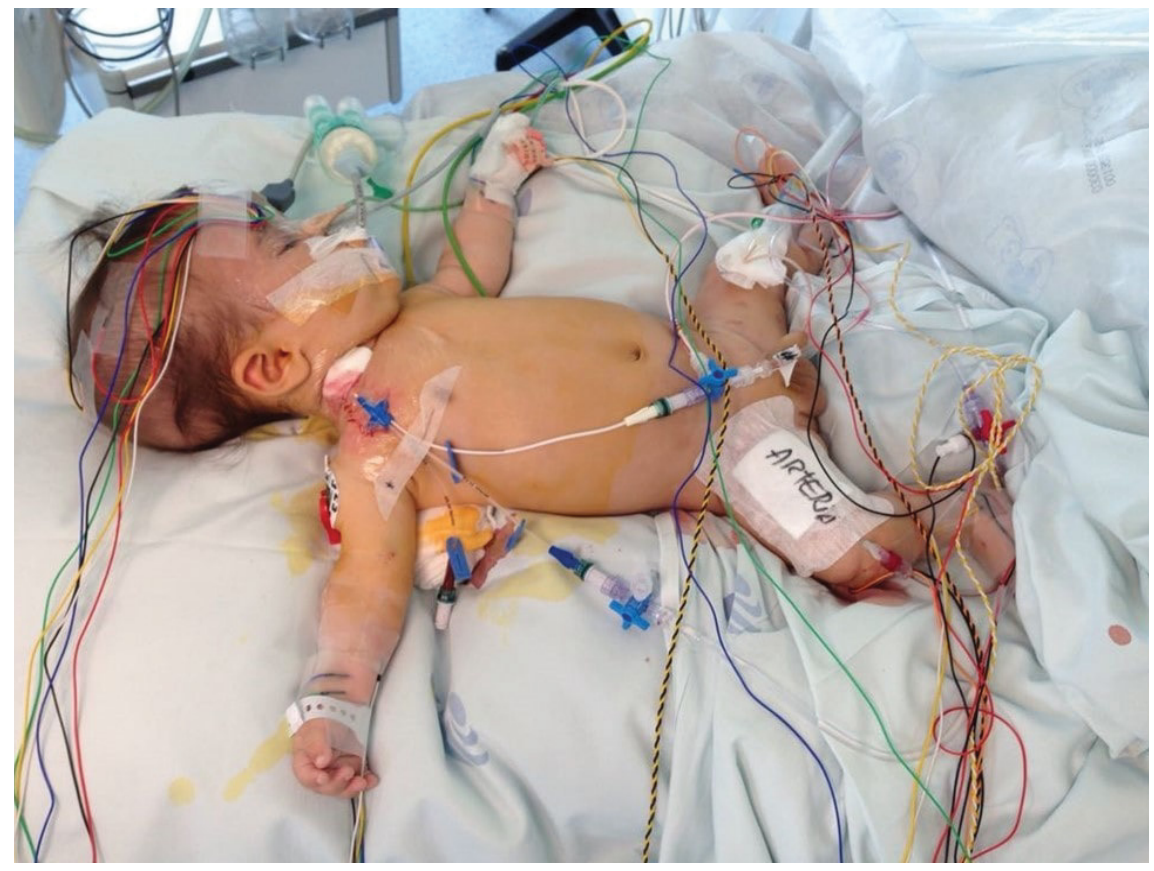

Figure 2: Immediate pre-positioning picture showing multimodal neuromonitoring.

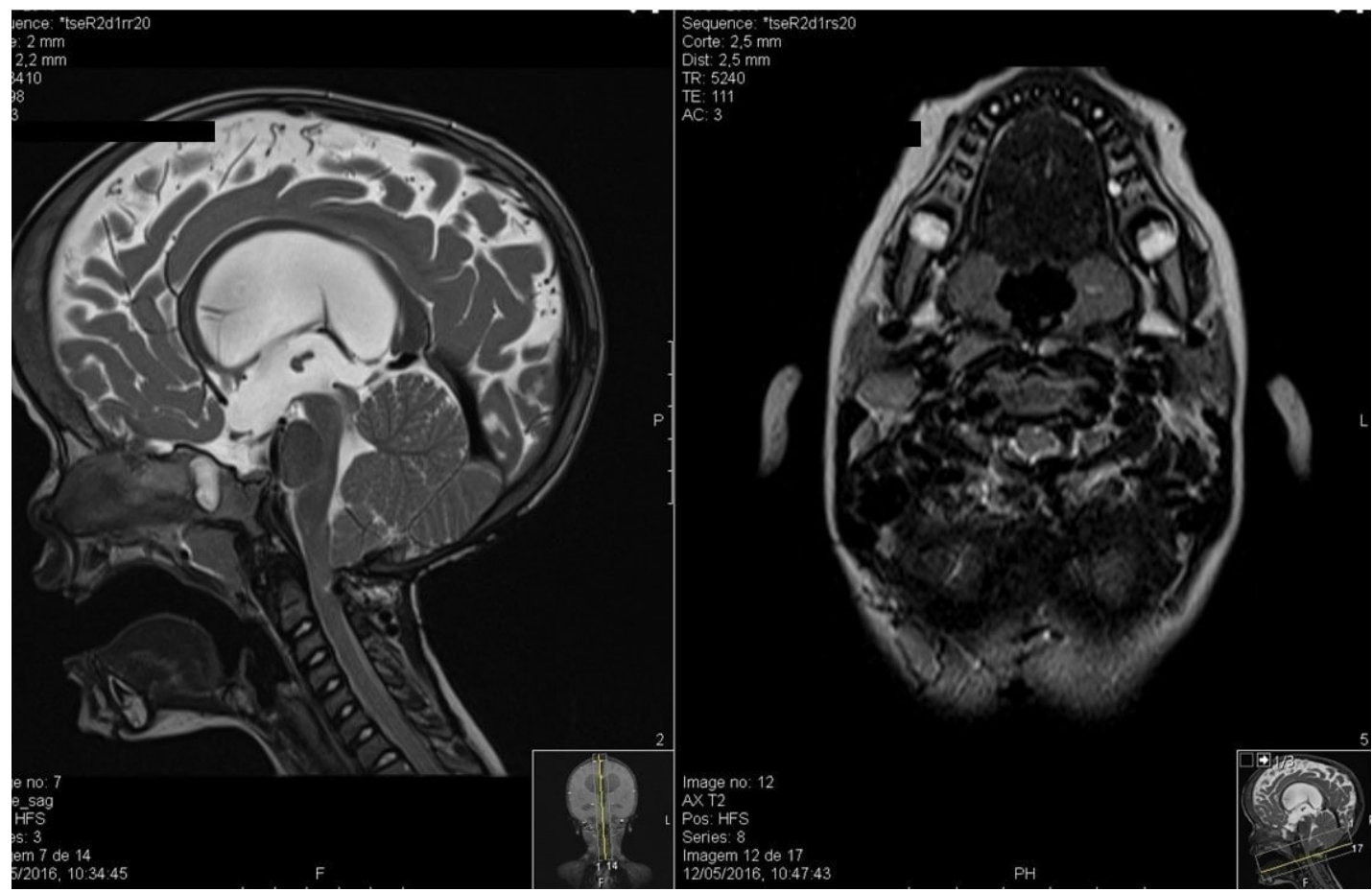

Figure 3: Postoperative MRI revealing a proper cervicomedullary decompression.

presented poor cephalic control, a cephalic perimeter crossing percentile lines upwards, and increased tension on the anterior fontanelle. Growth parameters of weight and length were harmonious and within the normal range not suggesting dwarfism/AChP. He had up-slanting palpebral fissures, a short neck and narrow thorax. He also had clinodactyly of the fifth finger in both hands and bilateral metatarsus adductus. Moreover, vertebral bodies malformation from Th8 to Th12 due to ossification centre defect causing hemivertebrae in Th10 and Th11 and, consequently, a scoliotic curve, were visible on skeleton X-rays. No relevant changes were found on long bones. Endocrine system workup was normal. He was referred to medical genetics. To present date no pathogenic variants explaining the phenotype have been identified. Transfontanellar ultrasound imaging showed hydrocephalus. Brain MRI confirmed hydrocephalus with periventricular oedema due to an obstruction of the CSF outflow from the 4th ventricle. Additionally, malformation of the craniovertebral transition was observed, with a short clivus, a small posterior fossa with a narrow foramen magnum, and 


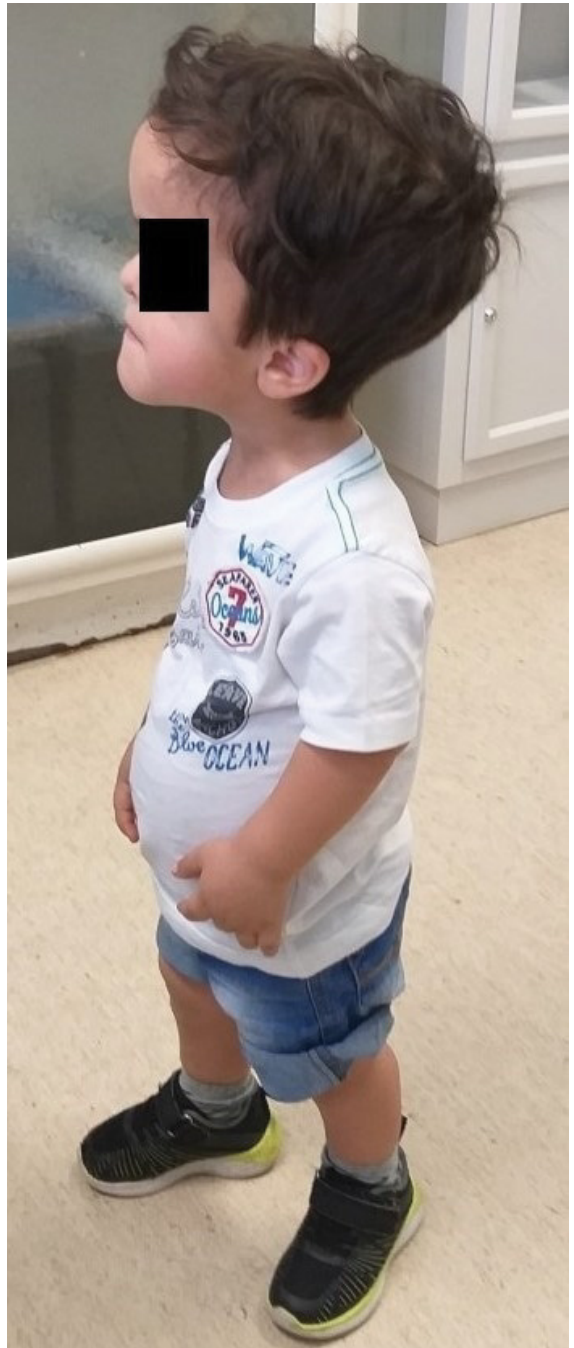

Figure 4: Picture of the patient after seven-years of follow-up.

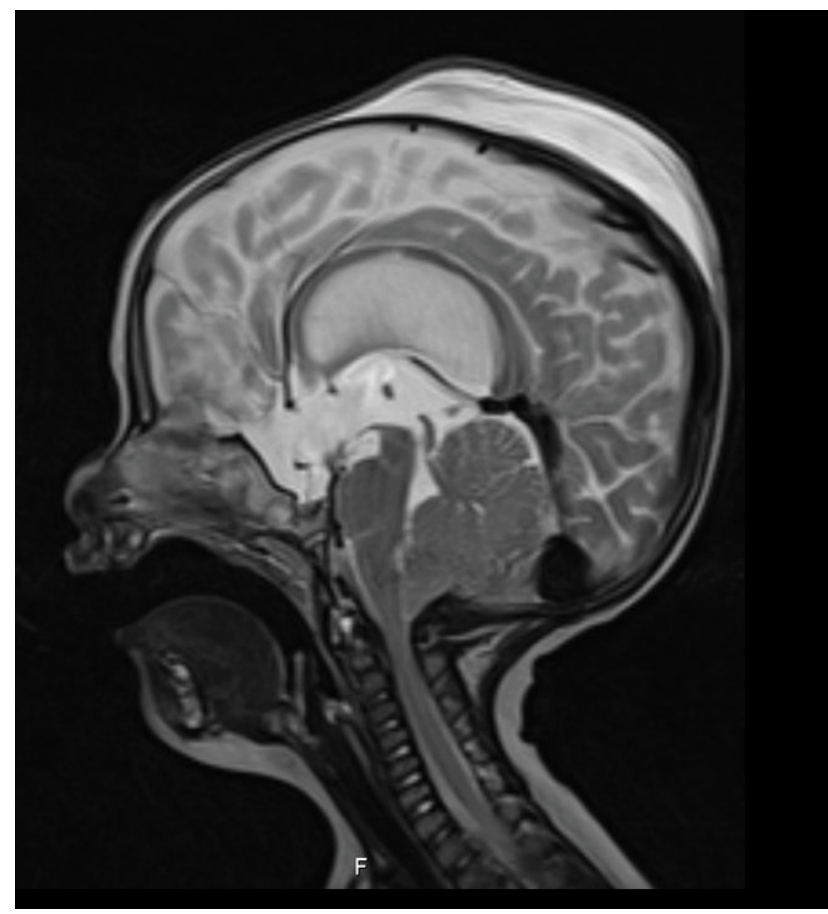

Figure 5: Patient 2-Sagittal plane MRI showing compression in the cervicomedullary transition (T2-weighted sequence). hypoplasia of the posterior atlas arch with marked cervicomedullary compression (Figure 5). An endoscopic third ventriculocisternostomy was successfully performed to treat hydrocephalus. The postoperative outcome was favourable with a stabilization of the cephalic perimeter and a normotensive anterior fontanelle. During follow-up, however, at one year of age, the child presented new-onset difficulty in cephalic control with a tendency to postural hyperextension, and difficulty in manipulating and controlling objects.

Craniocervical junction MRI with CSF flow study confirmed medullary compression with impaired CSF flow at the foramen magnum without $\mathrm{T} 2$ medullary hyperintensity. Craniovertebral decompressive surgery was performed - C1 laminectomy, posterior fossa craniectomy of approximately $2 \times 2$ centimetres and extensive release of fibrotic bands on the posterior atlanto-occipital membrane with dural expansion. Intraoperative ultrasound imaging with doppler showed adequate decompression of the cervicomedullary transition and an improvement in CSF flow velocities and hence no duraplasty was required. Multimodal intraoperative neuromonitoring was used to maximize surgical safety. The postoperative period was uneventful. During the 6 months of postoperative follow-up, the infant improved cephalic control and a boost in motor development has been observed. Follow-up MRI is illustrated in Figure 6.

\section{Discussion}

Cervicomedullary compression at craniovertebral junction due to foramen magnum malformation is common in patients with skeletal dysplasia, particularly those with AChP. In the majority of cases, it is asymptomatic [24]. However, when this cervicomedullary compression is relevant, significant potential complications can arise $[25,26]$. There is no strong evidence to support the proper management of cervicomedullary compression due to craniovertebral stenosis nor for the optimal time for intervention [17]. The skeletal dysplasia diagnosis is straightforward in AChP, however diagnosing other syndromes which include skeletal dysplasia can be extremely challenging. Genetic tests are becoming a particularly useful tool in clinical practice for diagnosing these syndromes, still, in about one-third of the cases no specific pathogenic variants are identified and there is yet a lot to learn regarding the natural course of this group of diseases [2]. Genetic test panels for skeletal dysplasia can hasten the proper diagnosis, which has important prognostic and management implications [27]. However, these tests are not always conclusive and clinical, laboratory and imaging data must be carefully interpreted to obtain a specific diagnosis, which might require several months or even years to cardinal features of a specific syndrome to be apparent. 


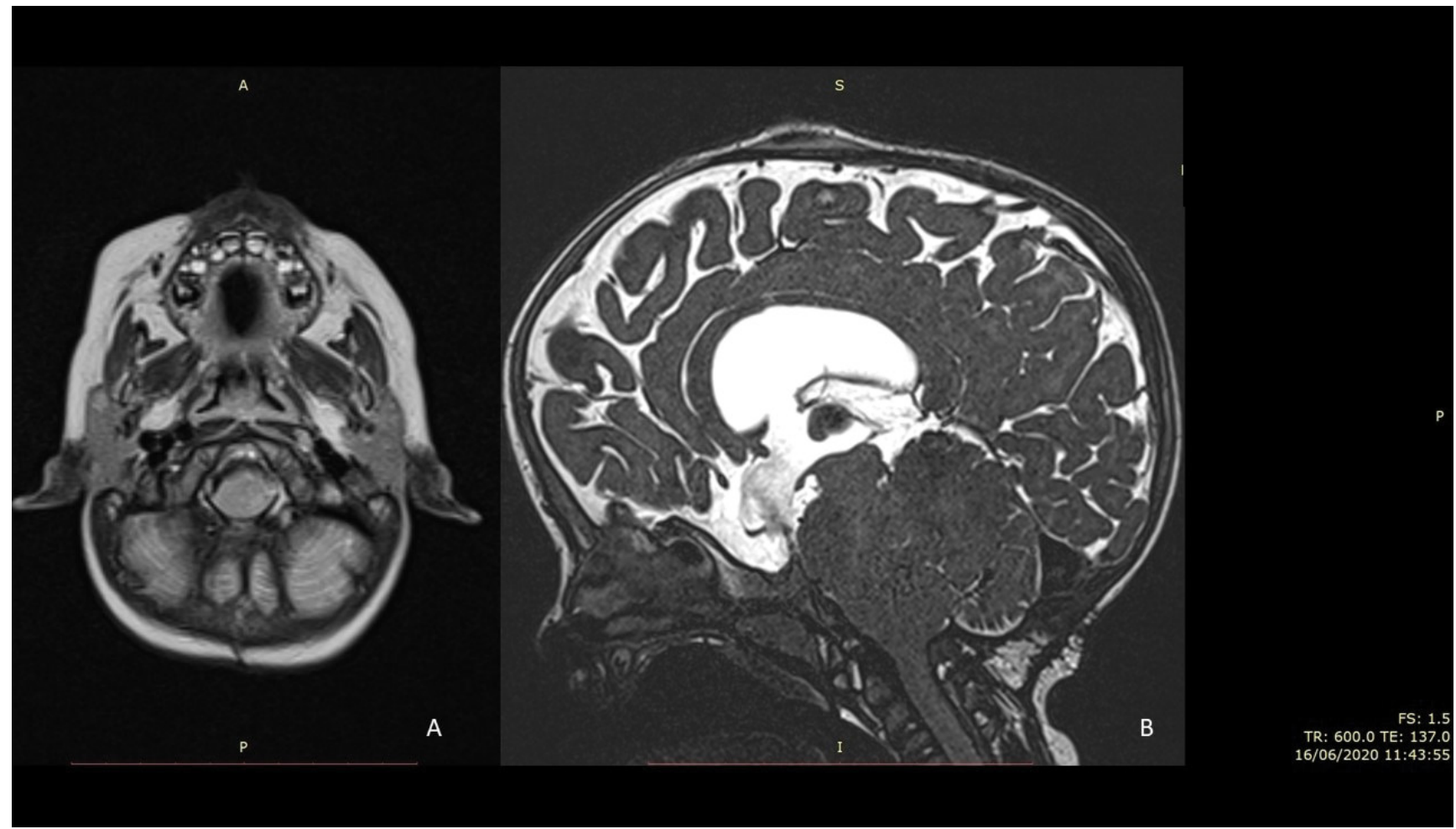

Figure 6: A) MRI T2 axial sequence trough foramen magnum revealing cervicomedullary decompression; B) MRI T2 Sagittal Constructive Interference in Steady State sequence revealing a C1 laminectomy and a suboccipital craniotomy decompressing the cervicomedullary transition. CSF flow through III ventricle ventriculocisternostomy can be appreciated.

In face of a craniovertebral stenosis, early decompressive surgery improves the deficits, as shown by Reid, et al. [8]. In fact, early decompression stops further neurological damage in the caudal brainstem and upper spinal cord and thus prevents myelopathy and respiratory impairment. Hence, surgical decompression should be performed as early as possible [17]. White K, et al. analysed the best practice procedures based on evidence and expert consensus about foramen magnum decompression in patients with AChP in the presence of clinical findings (delayed motor development, hypotonia, asymmetric use of the limbs), physical findings (clonus, motor weakness or spasticity, pathologic reflexes), test findings (disturbed sleep pattern with central events) and imaging findings such as an MRI demonstrating a narrow foramen magnum with indentation of the spinal cord (hyperintensity in T2-weighted sequences) - and determined treatment should be semi-urgent in such situations [7]. The same recommendation is stated in the 2020 guidelines for Achondroplasia [17]. Several studies have found in a consistent fashion that timely foramen magnum surgical decompression reduces mortality [28] and leads to a significant symptom improvement [19-23]. Surgical complications reported are nevertheless relevant and include CSF leakage, infection, pseudomeningocele and venous sinus haemorrhage [19-23]. Thus, a conservative attitude can be adopted in patients with foramen magnum stenosis with cervicomedullary compression but without MRI signal changes or neurological symptoms [17].
Decompressive surgery for craniovertebral stenosis is performed in $5 \%-25 \%$ of patients with AChP [20]. Craniovertebral decompressive surgery in very young infants, such as those reported, has some specificities that must be considered. It must be performed in specialized centres by experienced teams in paediatric and spine Neurosurgery and paediatric intensive care units to ensure close and appropriate surveillance in the postoperative period. Most frequently the chosen technique is a suboccipital craniectomy and C1 laminectomy $[19,20,22]$. We consider a meticulous and extensive dissection through the posterior atlantooccipital membrane releasing the fibrous bands usually found above the atlas in order to allow a proper dural expansion to be particularly important.

Since CSF leakage is the most common complication, especially in this group [19], we tend to avoid unnecessary duraplasty if there is an acceptable intraoperative dural expansion and if intraoperative ultrasound shows a good decompression of CSF spaces and CSF flow velocities improve through craniovertebral transition. Ultrasound application in Chiari malformation type I craniovertebral stenosis proved to be a reliable tool to decide if duraplasty was required, according to cerebrospinal flow velocities [29]. We believe that ultrasound B-mode and doppler effect can be useful to evaluate if an adequate decompression of neural structures is achieved also in foramen magnum stenosis. Some authors have already reported usage of intraoperative ultrasound in craniovertebral stenosis associated to skeletal dysplasia with good results 
[30,31]. Intraoperative multimodal neuro-monitoring is of paramount importance to maximize surgical safety and, in our opinion, it should be used right at the start of the intervention, from before patient prone positioning (to obtain basal values) until the end of the surgery. Neural compression can be severe in some cases, as in the patients we presented in this paper. In such cases, the simple positioning of the patient may cause severe and irreversible neural damage which can be prevented with multimodal neuromonitoring. Additionally, multimodal neuromonitoring may, intraoperatively, dictate that the initial surgical strategy must be changed in order to avoid further neural damage. Another important aspect provided by intraoperative monitoring concerns variation on motor and somatosensory evoked potentials' curves. Kawasaki Y, et al. have found improvement in the potentials' curves during decompression in Chiari type I malformation decompressive surgery as a good indicator for clinical recovery [32]. We believe that this knowledge could also be useful during skeletal $\mathrm{C} 0-\mathrm{C} 1$ decompression surgery from skeletal dysplasia with foramen magnum and upper cervical stenosis.

\section{Conclusion}

We present two rare cases with craniovertebral junction stenosis associated with skeletal dysplasia treated at a very young age with favourable outcomes. In infants with skeletal dysplasia, including AChP, a high clinical suspicion, as well as an early imaging and sleeping test diagnosis and referral to specialized centres, are crucial. Early surgical decompressive treatment, using intraoperative ultrasound and neurophysiologic monitoring, can improve the neurological outcome and survival.

\section{Statement of Ethics}

Informed consent for publications of patient photographs were obtained from infant parents. Informed consent was obtained from parents for submission of both case reports to this journal.

\section{Conflict of Interest Statement}

The authors declare that they have no conflict of interest.

\section{Funding Sources Statement}

This paper did not receive any specific grant from funding agencies in the public, commercial, or not-forprofit sectors.

\section{Authors' Contributions}

Cristiano Antunes designed and wrote the article and also participated in acquisition, analysis and interpretation of data; Rui Vaz and Daniela Alves made contributions writing the article and also participated in acquisition of data and critically revised the article; Josué Pereira and Paulo Pereira performed the surgeries and critically revised the article. All the authors approved the final version to be published.

\section{References}

1. Warman ML, Cormier-Daire V, Hall C, Krakow D, Lachman $R$, et al. (2011) Nosology and classification of genetic skeletal disorders: 2010 revision. Am J Med Genet A 155A: 943-968.

2. Krakow D (2015) Skeletal dysplasias. Clin Perinatol 42: 301-319.

3. Dutton RV (1987) A practical radiologic approach to skeletal dysplasias in infancy. Radiol Clin North Am 25: 1211-1233.

4. Dugherty A (2017) Achondroplasia: Etiology, clinical presentation, and management. Neonatal Netw 36: 337342.

5. Carter EM, Davis JG, Raggio CL (2007) Advances in understanding etiology of achondroplasia and review of management. Curr Opin Pediatr 19: 32-37.

6. Marin-Padilla M, Marin-Padilla TM (1977) Developmental abnormalities of the occipital bone in human chondrodystrophies (achondroplasia and thanatophoric dwarfism). Birth Defects Orig Artic Ser 13: 7-23.

7. White KK, Bompadre V, Goldberg MJ, Bober MB, Campbell $\mathrm{JW}$, et al. (2016) Best practices in the evaluation and treatment of foramen magnum stenosis in achondroplasia during infancy. Am J Med Genet A 170A: 42-51.

8. Reid CS, Pyeritz RE, Kopits SE, Maria BL, Wang $\mathrm{H}$, et al. (1987) Cervicomedullary compression in young patients with achondroplasia: Value of comprehensive neurologic and respiratory evaluation. J Pediatr 110: 522-530.

9. Pauli RM (2019) Achondroplasia: A comprehensive clinical review. Orphanet J Rare Dis 14: 1.

10. Hecht JT, Bodensteiner JB, Butler IJ (2014) Neurologic manifestations of achondroplasia. Handb Clin Neurol 119: 551-563.

11. Simmons K, Hashmi SS, Scheuerle A, Canfield M, Hecht JT (2014) Mortality in babies with achondroplasia: Revisited. Birth Defects Res A Clin Mol Teratol 100: 247-249.

12. Nadel JL, Wilkinson DA, Garton HJL, Muraszko KM, Maher CO (2018) Screening and surgery for foramen magnum stenosis in children with achondroplasia: A large, national database analysis. J Neurosurg Pediatr 23: 374-380.

13. Trotter TL, Hall JG, American Academy of Pediatrics Committee on $\mathrm{G}$ (2005) Health supervision for children with achondroplasia. Pediatrics 116: 771-783.

14. Bosemani T, Orman G, Carson KA, Meoded A, Huisman TA, et al. (2014) Diffusion tensor imaging of the brainstem in children with achondroplasia. Dev Med Child Neurol 56: 1085-1092.

15. Colamaria V, Mazza C, Beltramello A, Polo A, Boner A, et al. (1991) Irreversible respiratory failure in an achondroplastic child: The importance of an early cervicomedullary decompression, and a review of the literature. Brain Dev 13: $270-279$.

16. Mador MJ, Tobin MJ (1990) Apneustic breathing. A characteristic feature of brainstem compression in achondroplasia? Chest 97: 877-883.

17. Kubota T, Adachi M, Kitaoka T, Hasegawa K, Ohata $Y$, et al. (2020) Clinical practice guidelines for achondroplasia. Clin Pediatr Endocrinol 29: 25-42. 
18. Aryanpur J, Hurko O, Francomano C, Wang H, Carson B (1990) Craniocervical decompression for cervicomedullary compression in pediatric patients with achondroplasia. $J$ Neurosurg 73: 375-382.

19. Bagley CA, Pindrik JA, Bookland MJ, Camara-Quintana $J Q$, Carson BS (2006) Cervicomedullary decompression for foramen magnum stenosis in achondroplasia. J Neurosurg 104: 166-172.

20. King JA, Vachhrajani S, Drake JM, Rutka JT (2009) Neurosurgical implications of achondroplasia. J Neurosurg Pediatr 4: 297-306.

21. Pauli RM, Horton VK, Glinski LP, Reiser CA (1995) Prospective assessment of risks for cervicomedullaryjunction compression in infants with achondroplasia. Am J Hum Genet 56: 732-744.

22. Reina V, Baujat G, Fauroux B, Couloigner V, Boulanger $E$, et al. (2014) Craniovertebral junction anomalies in achondroplastic children. Adv Tech Stand Neurosurg 40: 295-312.

23. Shimony N, Ben-Sira L, Sivan Y, Constantini S, Roth J (2015) Surgical treatment for cervicomedullary compression among infants with achondroplasia. Childs Nerv Syst 31: 743-750.

24. Ireland PJ, Pacey V, Zankl A, Edwards P, Johnston LM, et al. (2014) Optimal management of complications associated with achondroplasia. Appl Clin Genet 7: 117-125.
25. Pauli RM, Scott CI, Wassman ER, Jr., Gilbert EF, Leavitt LA, et al. (1984) Apnea and sudden unexpected death in infants with achondroplasia. J Pediatr 104: 342-348.

26. Rimoin DL (1995) Cervicomedullary junction compression in infants with achondroplasia: When to perform neurosurgical decompression. Am J Hum Genet 56: 824-827.

27. Nikkel SM (2017) Skeletal dysplasias: What every bone health clinician needs to know. Curr Osteoporos Rep 15: 419-424.

28. Ho NC, Guarnieri M, Brant LJ, Park SS, Sun B, et al. (2004) Living with achondroplasia: Quality of life evaluation following cervico-medullary decompression. Am J Med Genet A 131: 163-167.

29. Brock RS, Taricco MA, de Oliveira MF, de Lima Oliveira M, Teixeira MJ, et al. (2017) Intraoperative ultrasonography for definition of less invasive surgical technique in patients with chiari type i malformation. World Neurosurg 101: 466-475.

30. Ryken TC, Menezes AH (1994) Cervicomedullary compression in achondroplasia. J Neurosurg 81: 43-48.

31. Yundt KD, Park TS, Tantuwaya VS, Kaufman BA (1996) Posterior fossa decompression without duraplasty in infants and young children for treatment of Chiari malformation and achondroplasia. Pediatr Neurosurg 25: 221-226.

32. Kawasaki Y, Uchida S, Onishi K, Toyokuni M, Okanari K, et al. (2017) Intraoperative neurophysiologic monitoring for prediction of postoperative neurological improvement in a child with chiari type i malformation. J Craniofac Surg 28: 1837-1841. 